\title{
Hunting for Dynamical Supersymmetry Breaking in Theories That S-confine
}

\author{
Tongyan $\operatorname{Lin}^{a, 1}$ John D. MASON ${ }^{a, 2}$ And AqIL SAJJAD ${ }^{a, 3}$ \\ ${ }^{a}$ Jefferson Physical Laboratory, Harvard University, Cambridge, Massachusetts 02138, USA
}

November 8, 2018

\begin{abstract}
The s-confining theories are a class of supersymmetric gauge theories with infrared dynamics which are well-understood. Perturbing such theories can give rise to dynamical supersymmetry breaking. We realize simple models of dynamical supersymmetry breaking by perturbing two of the $10 S U(N)$ s-confining gauge theories by a single trilinear operator. These examples have locally stable vacua with spontaneous supersymmetry breaking. The first is $S U(5)$ with two generations (consisting of an antisymmetric tensor and an antifundamental) plus two flavors. The second is $S U(5)$ with three generations. The properties of the former vacuum are calculable while those of the latter vacuum are not. We briefly discuss the other $S U(N)$ models.
\end{abstract}

\footnotetext{
${ }^{1}$ E-mail: tongyan@physics.harvard.edu

2 E-mail: jdmason@physics.harvard.edu

${ }^{3}$ E-mail: sajjad@physics.harvard.edu
} 


\section{INTRODUCTION AND OVERVIEW}

Supersymmetry (SUSY) breaking at low scales is an attractive solution to the hierarchy problem. A compelling explanation for the origin of such low scales is dynamical supersymmetry breaking (DSB), where small mass scales arise from dimensional transmutation: $\Lambda=M_{p l} \exp \left(\frac{-8 \pi^{2}}{g^{2}\left(M_{p l}\right)}\right)$ [1]. Understanding theories that exhibit this structure requires computing non-perturbative or strong gauge dynamics, which is in general very challenging.

Supersymmetric gauge theories with a weakly coupled magnetic dual provide a tractable framework for building models of dynamical supersymmetry breaking. In such theories, the effective description of the infrared physics is severely constrained by global symmetries and by the holomorphy of the superpotential. In particular, this technique was used to understand vector-like $\mathcal{N}=1$ theories [2-6] and later useful for understanding $\mathcal{N}=1$ models with vector-like matter and one antisymmetric tensor [7-11]. Deforming these theories with $\delta W \neq 0$ can lead to models of DSB, where the confining dynamics generates a weakly coupled O'Raifeartaigh model [12]. These models typically possess an R-symmetry [13] and relevant operators with mass scales $m \ll M_{p l}$. For example, adding a tree-level mass perturbation, $\delta W=m \operatorname{Tr}(\bar{Q} Q)$, to vector-like $S U\left(N_{c}\right)$ supersymmetric QCD with $N_{c}<N_{F}<\frac{3}{2} N_{c}$ flavors is sufficient to break supersymmetry in a vacuum that is meta-stable but parametrically long lived [14]. The O'Raifeartaigh model has an approximate R-symmetry, and the hierarchically small mass scale is given by $\sqrt{m \Lambda}$.

It is important to ask if other types of O'Raifeartaigh models can emerge from a richer set of gauge dynamics. Happily, there exists a special class of supersymmetric theories that exhibit confining dynamics and are well understood, the so-called "s-confining" theories. In these theories, the moduli space has a smooth description in terms of gauge invariant composites everywhere, including the origin $[15,16]$. The composites generally obey classical constraint equations, which are realized in the IR theory by the presence of a dynamical superpotential. The classic example of an s-confining theory is the case of $N_{F}=N_{c}+1$ in the vector-like $S U\left(N_{c}\right)$ gauge theories mentioned above. The gauge invariant composites are the $N_{F}^{2}$ mesons, $M^{i j}=\bar{Q}^{i} Q^{j}$, the $N_{F}$ baryons, $B^{i}=\epsilon Q^{N_{c}}$, and the $N_{F}$ anti-baryons, $\bar{B}^{i}=\epsilon \bar{Q}^{N_{c}}[5]$. Below the confinement scale $\Lambda$, the theory admits a description in terms of the composites and a dynamical superpotential:

$$
W^{d y n}=\frac{1}{\Lambda^{2 N_{c}-1}}\left(\bar{B}^{i} M^{i j} B^{j}-\operatorname{det} M\right) .
$$




\begin{tabular}{|l|ll|}
\hline Theory 1 & $S U(N)$ & $(N+1)(\square+\bar{\square})$ \\
Theory 2 & $S U(2 N)$ & $\boxminus+2 N \bar{\square}+4 \square$ \\
Theory 3 & $S U(2 N+1)$ & $\boxminus+(2 N+1) \bar{\square}+4 \square$ \\
Theory 4 & $S U(2 N+1)$ & $\boxminus+\bar{\theta}+3(\square+\bar{\square})$ \\
Theory 5 & $S U(2 N)$ & $\boxminus+\bar{\theta}+3(\square+\bar{\square})$ \\
Theory 6 & $S U(6)$ & $\boxminus+4(\square+\bar{\square})$ \\
Theory 7 & $S U(5)$ & $3(\boxminus+\bar{\square})$ \\
Theory 8 & $S U(5)$ & $2 \boxminus+2 \square+4 \bar{\square}$ \\
Theory 9 & $S U(6)$ & $2 \boxminus+5 \bar{\square}+\square$ \\
Theory 10 & $S U(7)$ & $2(\boxminus+3 \bar{\square})$ \\
\hline
\end{tabular}

TABLE I. s-confining $S U(N)$ theories [16].

As described above, a mass perturbation will lead to supersymmetry breaking in this theory.

In this paper, we search for dynamical supersymmetry breaking vacua by adding simple perturbations $(\delta W \neq 0)$ to the $S U(N)$ s-confining theories. See [17-20] for related investigations of some of the s-confining theories. A complete list of the $S U(N)$ theories (as well as the $S O(N)$ and $S p(N)$ theories) was found in [16], and is given in Table I. Our criteria for a simple perturbation is the single lowest dimension operator allowed by gauge invariance, where we will include weakly gauged global symmetries in our analysis in one case. We generally find three qualitatively different behaviors for models obeying our criteria. First, most models possess no supersymmetry breaking vacua, even locally. Second, some models have calculable, locally stable, and supersymmetry-breaking vacua at small field vacuum expectation values $(\mathrm{VEVs})$. These vacua are parametrically long lived and calculable, as in [14]. Third, some models realize dynamical supersymmetry breaking, but in a vacuum with field VEVs of order $\mathcal{O}(\Lambda)$; a controlled calculation of the properties of the vacuum is not possible because the Kähler potential is not known. However, one can conclude that supersymmetry is broken based on the knowledge of the superpotential.

According to our criteria, most of the perturbed $S U(N)$ s-confining theories do not break supersymmetry or are incalculable. We therefore focus our analysis on two of the 10 models; our numbering of the theories follows the convention of [16]. Note that theory 1 is precisely the s-confining example given above [14] and theory 6 is studied in [19]. In Section II, we 


\begin{tabular}{c|c|cccccc} 
& $S U(5)$ & $S U(2)_{1}$ & $S U(4)$ & $S U(2)_{2}$ & $U(1)_{1}$ & $U(1)_{2}$ & $U(1)_{R}$ \\
\hline$A$ & $\boxminus$ & $\square$ & 1 & 1 & 0 & -1 & 0 \\
$\bar{Q}$ & $\square$ & 1 & $\square$ & 1 & 1 & 1 & $\frac{1}{3}$ \\
$Q$ & $\square$ & 1 & 1 & $\square$ & -2 & 1 & $\frac{1}{3}$ \\
\hline \hline$P_{1}=Q \bar{Q}$ & & 1 & $\square$ & $\square$ & -1 & 2 & $\frac{2}{3}$ \\
$X=A \bar{Q}^{2}$ & & $\square$ & $\boxminus$ & 1 & 2 & 1 & $\frac{2}{3}$ \\
$P_{2}=A^{2} Q$ & & $\square$ & 1 & $\square$ & -2 & -1 & $\frac{1}{3}$ \\
$Y=A^{3} \bar{Q}$ & & $\square$ & $\square$ & 1 & 1 & -2 & $\frac{1}{3}$ \\
$Z=A^{2} Q^{2} \bar{Q}$ & & 1 & $\square$ & 1 & -3 & 1 & 1
\end{tabular}

TABLE II. The matter content and symmetries of Theory 8, an $S U(5)$ gauge theory. $A, \bar{Q}$ and $Q$ are the microscopic degrees of freedom, and the other fields are the composites of the IR theory.

study the effect of a trilinear perturbation to theory 8, an $S U(5)$ gauge theory with two antisymmetric tensors, two fundamental fields, and 4 antifundamental fields. A calculable and meta-stable supersymmetry breaking vacuum exists near the origin of field space, and the $\mathrm{R}$ symmetry is unbroken. In Section III we analyze theory 7, an $S U(5)$ gauge theory with three generations of an antisymmetric tensor and an antifundamental field. Adding the simplest trilinear perturbation gives rise to supersymmetry breaking, but with a runaway direction. At large field VEVs, higher-dimension perturbations can lift all flat directions, yielding a supersymmetry breaking vacuum in an uncalculable regime. The approximate R-symmetry is spontaneously broken. In Section IV, we briefly discuss perturbations of the other $S U(N)$ s-confining theories.

\section{THEORY 8 - SU(5) WITH $2 \boxminus+4 \bar{\square}+2 \square$}

Theory 8 has gauge group and matter content: $S U(5)$ with $2 \boxminus+4 \bar{\square}+2 \square$, where $\mathrm{G}$ is an antisymmetric tensor, $A$; $\bar{\square}$ is an anti-fundamental, $\bar{Q}$; and $\square$ is a fundamental, $Q$. When $W=0$, the theory has an anomaly-free $S U(2)_{1} \times S U(4) \times S U(2)_{2} \times U(1)_{1} \times U(1)_{2} \times U(1)_{R}$ global symmetry, with the charges given in Table II.

In the strongly coupled IR regime, the theory has an s-confined description in terms of 
five gauge invariant degrees of freedom: $P_{1}=\bar{Q} Q, X=A \bar{Q}^{2}, P_{2}=A^{2} Q, Y=A^{3} \bar{Q}, Z=$ $A^{2} Q^{2} \bar{Q}^{2}$. The global symmetry is unbroken, and there is a dynamical superpotential which enforces the classical constraints[16, 18]:

$$
W^{(d y n)}=\frac{1}{\Lambda^{9}}\left(3 X Y Z-P_{1}^{2} Y^{2}+3 P_{1} P_{2} X Y-\frac{9}{32} P_{2}^{2} X^{2}\right) .
$$

We consider perturbing the $W=0$ theory by the gauge invariant operator $A \bar{Q}^{2}$ :

$$
\delta W=\lambda J_{i a b}\left[A \bar{Q}^{2}\right]^{i a b}
$$

where

$$
J_{i a b}=\frac{1}{\sqrt{2}}\left[\left(\begin{array}{cccc}
0 & 1 & 0 & 0 \\
-1 & 0 & 0 & 0 \\
0 & 0 & 0 & 1 \\
0 & 0 & -1 & 0
\end{array}\right),\left(\begin{array}{cccc}
0 & 1 & 0 & 0 \\
-1 & 0 & 0 & 0 \\
0 & 0 & 0 & 1 \\
0 & 0 & -1 & 0
\end{array}\right)\right] \text {, }
$$

and $i \in S U(2)_{1}, a, b \in S U(4)$. This choice of perturbation preserves an $S p(4) \times S U(2)_{2} \times$ $U(1) \times U(1)_{R}$ symmetry, where $S p(4) \subset S U(4)$. Sitting inside these groups are four anomaly free global $U(1)$ 's. Gauging a linear combination of these is sufficient to forbid all gaugeinvariant operators up to and including dimension 3, except for the non-zero terms appearing in Eq. (3). (This gauged $U(1)$ will also play an important role in Section II B when we consider the fate of the fields $P_{1}$ and $P_{2}$.) Thus other allowed operators are higher dimensional in the elementary description and suppressed by $\mathcal{O}\left(\frac{1}{M_{*}^{n}}\right)$, where $n$ is a positive integer and $M_{*}$ is some high scale. We work in the limit that $\frac{\Lambda}{M_{*}} \ll \lambda \ll 1$, in which case we can treat the operator in Eq. (3) as a perturbation and neglect all other gauge-invariant operators.

The s-confined description of the perturbed theory is then:

$$
W=\frac{1}{\Lambda^{9}}\left(3 X Y Z-P_{1}^{2} Y^{2}+3 P_{1} P_{2} X Y-\frac{9}{32} P_{2}^{2} X^{2}\right)+\lambda X .
$$

The Kähler potential is $K=\frac{1}{\alpha_{P_{1}}|\Lambda|^{2}} P_{1}^{\dagger} P_{1}+\frac{1}{\alpha_{X}|\Lambda|^{4}} X^{\dagger} X+\frac{1}{\alpha_{P_{2}}|\Lambda|^{4}} P_{2}^{\dagger} P_{2}+\frac{1}{\alpha_{Y}|\Lambda|^{6}} Y^{\dagger} Y+\frac{1}{\alpha_{Z}|\Lambda|^{8}} Z^{\dagger} Z$, up to terms $\mathcal{O}\left(\phi^{\dagger} \phi \phi^{\dagger} \phi\right)$ where $\alpha_{\phi}>0$ and $\phi=X, Y, Z, P_{1}, P_{2}$. These higher order terms become negligible when studying the theory near the origin of the moduli space, where $|\phi| \ll \Lambda$. Rescaling the fields for canonical kinetic terms, we have

$$
\begin{aligned}
W & =3 \sqrt{\alpha_{X} \alpha_{Y} \alpha_{Z}} X Y Z+\lambda \sqrt{\alpha_{X}} \Lambda^{2} X \\
& +\frac{1}{\Lambda}\left(-\alpha_{P_{1}} \alpha_{Y} P_{1}^{2} Y^{2}+3 \sqrt{\alpha_{P_{1}} \alpha_{P_{2}} \alpha_{X} \alpha_{Y}} P_{1} P_{2} X Y-\frac{9}{32} \alpha_{P_{2}} \alpha_{X} P_{2}^{2} X^{2}\right) .
\end{aligned}
$$


The second line of the above equation consists of non-renormalizable terms suppressed by $\Lambda$; these terms can be neglected if $|\phi| \ll \Lambda$. We will return to this point later. Rewriting $h=3 \sqrt{\alpha_{X} \alpha_{Y} \alpha_{Z}}$ and $F=\frac{\lambda \Lambda^{2}}{3 \sqrt{\alpha_{Y} \alpha_{Z}}}$, and putting in the explicit flavor indices, the effective description of the theory near the origin becomes:

$$
W=h\left(\epsilon_{i j} \epsilon_{a b c d} X^{i a b} Y^{j c} Z^{d}+F J_{i a b} X^{i a b}\right) .
$$

We now show that near the origin, this theory admits a locally stable supersymmetry breaking vacuum. We will show that supersymmetry is spontaneously broken, parameterize the moduli space of the theory, choose a vacuum about which to expand, and compute the 1-loop correction to pseudomoduli masses. We show that the fields $P_{1,2}$ are also stabilized below $\Lambda$ due to the presence of the weakly gauged flavor symmetry. This will suffice to show that our choice of vacuum is locally stable. We then briefly address the metastability of the vacuum.

First, this theory has no supersymmetric vacuum due to a rank-breaking condition [14]. The F-term equations for the field $X$ are:

$$
\left(F_{X}\right)_{1 a b}=h F J_{1 a b}+h \epsilon_{a b c d} Y^{2 c} Z^{d},\left(F_{X}\right)_{2 a b}=h F J_{2 a b}-h \epsilon_{a b c d} Y^{1 c} Z^{d} .
$$

Here $J_{1 a b}$ and $J_{2 a b}$ are rank-4 antisymmetric matrices. However, $\epsilon_{a b c d} Y^{2 c} Z^{d}$ is the antisymmetric outer product of two vectors, $Y^{2 c}$ and $Z^{d}$, which has a rank of at most 2 . Therefore there is no solution to the $F_{X}$ equations and this is an example of rank-breaking. Furthermore, there is no runaway direction with arbitrarily small potential energy.

We can find a vacuum in this theory by choosing field VEVs that cancel off as many F-terms as possible, thereby minimizing the vacuum energy. Since the rank of the antisymmetrized outer product of the $Y$ and $Z$ vectors is at most two, we can set two of the F-term equations to zero by giving VEVs to the $Y$ and $Z$ fields. We have found solutions by replacing $J_{i a b} \rightarrow J_{i a b}^{\prime}$ where

$$
J_{i a b}^{\prime}=\frac{1}{\sqrt{2}}\left[\left(\begin{array}{cccc}
0 & 1 & 0 & 0 \\
-1 & 0 & 0 & 0 \\
0 & 0 & 0 & 0 \\
0 & 0 & 0 & 0
\end{array}\right),\left(\begin{array}{cccc}
0 & 1 & 0 & 0 \\
-1 & 0 & 0 & 0 \\
0 & 0 & 0 & 0 \\
0 & 0 & 0 & 0
\end{array}\right)\right] .
$$

The solutions to these modified F-term equations give the minimum-energy field configuration in the full supersymmetry breaking theory. 
Before we describe the fields VEVs in our vacuum, it will be convenient to parameterize the fields $X, Y, Z$ in the following way. Writing the field $X$ in terms of its $S U(2)_{1}$ components, $X^{i}=\left(X^{1}, X^{2}\right)$, then we can define new fields

$$
X^{A} \equiv \frac{1}{\sqrt{2}}\left(X^{2}-X^{1}\right), X^{S} \equiv \frac{1}{\sqrt{2}}\left(X^{1}+X^{2}\right),
$$

where the fields $X^{A}$ and $X^{S}$ are

$$
X^{S} \equiv\left(\begin{array}{cccc}
0 & x_{12}^{S} & x_{13}^{S} & x_{14}^{S} \\
-x_{12}^{S} & 0 & x_{23}^{S} & x_{24}^{S} \\
-x_{13}^{S} & -x_{23}^{S} & 0 & x_{34}^{S} \\
-x_{14}^{S} & -x_{24}^{S} & -x_{34}^{S} & 0
\end{array}\right), \quad X^{A} \equiv\left(\begin{array}{cccc}
0 & x_{12}^{A} & x_{13}^{A} & x_{14}^{A} \\
-x_{12}^{A} & 0 & x_{23}^{A} & x_{24}^{A} \\
-x_{13}^{A} & -x_{23}^{A} & 0 & x_{34}^{A} \\
-x_{14}^{A} & -x_{24}^{A} & -x_{34}^{A} & 0
\end{array}\right),
$$

so that $X^{i}=\left(\left(X^{S}-X^{A}\right),\left(X^{S}+X^{A}\right)\right) / \sqrt{2}$. We make a similar field redefinition for the $Y$-fields: $Y^{i}=\left(Y^{1}, Y^{2}\right), Y^{A} \equiv\left(Y^{2}-Y^{1}\right) / \sqrt{2}$, and $Y^{S} \equiv\left(Y^{1}+Y^{2}\right) / \sqrt{2}$, with

$$
\begin{gathered}
Y^{S} \equiv\left(\begin{array}{llll}
y_{1}^{S} & y_{2}^{S} & y_{3}^{S} & y_{4}^{S}
\end{array}\right), \quad Y^{A} \equiv\left(\begin{array}{llll}
y_{1}^{A} & y_{2}^{A} & y_{3}^{A} & y_{4}^{A}
\end{array}\right), \\
Y \equiv \frac{1}{\sqrt{2}}\left(\left(Y^{S}-Y^{A}\right),\left(Y^{S}+Y^{A}\right)\right) .
\end{gathered}
$$

Finally, the $Z$ field is

$$
Z=\left(\begin{array}{llll}
z_{1} & z_{2} & z_{3} & z_{4}
\end{array}\right)
$$

The solution that minimizes the potential $V$ is

$$
\begin{gathered}
z_{1}=z_{2}=y_{1}^{A}=y_{2}^{A}=y_{1}^{S}=y_{2}^{S}=x_{12}^{S}=x_{13}^{S}=x_{14}^{S}=x_{23}^{S}=x_{24}^{S}=x_{12}^{A}=0, \\
\frac{x_{13}^{A}}{x_{14}^{A}}=\frac{x_{23}^{A}}{x_{24}^{A}}=\frac{y_{3}^{S}}{y_{4}^{S}}=\frac{z_{3}}{z_{4}} \quad \text { and } \quad y_{3}^{A}=-\frac{1}{z_{4}}\left(F-y_{4}^{A} z_{3}\right) .
\end{gathered}
$$

This set of equations can be generated by computing the Gröbner basis of the modified F-term equations from Eq. (9). The Gröbner basis is a generating set for the ideal corresponding to a set of polynomial equations [21, 22], in this case the F-term equations. We used the Gröbner basis algorithm implemented in Mathematica.

We can use these equations to eliminate all variables but 8 . These 8 complex variables span the classical moduli space:

$$
z_{3}, z_{4}, y_{4}^{S}, y_{4}^{A}, x_{14}^{A}, x_{24}^{A}, x_{34}^{A}, x_{34}^{S}
$$

Therefore the classical vacuum structure of the renormalizable IR theory, with the full perturbation to the superpotential, includes an 8-dimensional moduli space of vacua with the minimum potential energy $V=2|h F|^{2}$. 
At an arbitrary point in the moduli space, the fields are

$$
\begin{aligned}
& X^{A}=\left(\begin{array}{cccc}
0 & 0 & x_{14}^{A} \frac{z_{3}}{z_{4}} & x_{14}^{A} \\
0 & 0 & x_{24}^{A} \frac{z_{3}}{z_{4}} & x_{24}^{A} \\
-x_{14}^{A} \frac{z_{3}}{z_{4}} & -x_{24}^{A} \frac{z_{3}}{z_{4}} & 0 & x_{34}^{A} \\
-x_{14}^{A} & -x_{24}^{A} & -x_{34}^{A} & 0
\end{array}\right), X^{S}=\left(\begin{array}{cccc}
0 & 0 & 0 & 0 \\
0 & 0 & 0 & 0 \\
0 & 0 & 0 & x_{34}^{S} \\
0 & 0 & -x_{34}^{S} & 0
\end{array}\right),
\end{aligned}
$$

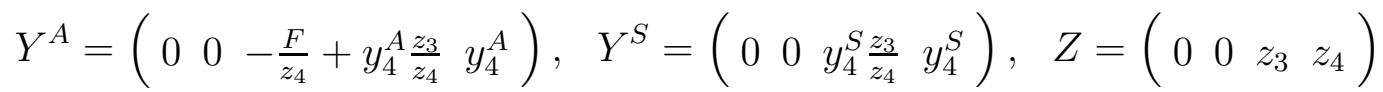

We pick a simple vacuum to expand about, with $z_{4}=\sqrt{F}$ and all other moduli set to zero:

$$
X=0, \quad Y=\sqrt{\frac{F}{2}}\left(\begin{array}{cccc}
0 & 0 & 1 & 0 \\
0 & 0 & -1 & 0
\end{array}\right), \quad Z=\left(\begin{array}{llll}
0 & 0 & 0 & \sqrt{F}
\end{array}\right)
$$

The light fields in this vacuum are either moduli or Goldstone bosons, which we distinguish by examining the symmetries broken in this vacuum. The $U(1)_{R}$ is unbroken because $X=0$. For the remaining $U(1) \times S p(4)$ symmetry, 4 linear combinations of the 11 generators are preserved by the $Y$ and $Z$ VEVs, leaving 7 Goldstone bosons:

$$
\begin{gathered}
\operatorname{Im}\left(y_{1}^{A}-\bar{z}_{2}\right), \operatorname{Re}\left(\bar{y}_{1}^{A}-z_{2}\right), \operatorname{Im}\left(y_{2}^{A}+\bar{z}_{1}\right), \operatorname{Re}\left(\bar{y}_{2}^{A}+z_{1}\right), \\
\operatorname{Im}\left(y_{4}^{A}+\bar{z}_{3}\right), \operatorname{Re}\left(\bar{y}_{4}^{A}+z_{3}\right), \operatorname{Im}\left(y_{3}^{A}+z_{4}\right) .
\end{gathered}
$$

As can be seen from the last 3 fields, three of the Goldstone bosons mix with the moduli, in particular $y_{4}^{A}, z_{3}, z_{4}$. So the 16 classical moduli split into 13 pseudomoduli, which receive 1loop masses from supersymmetry breaking, and 3 Goldstone bosons, which remain massless.

\section{A. One-loop masses}

We now evaluate the pseudomoduli masses at one-loop order and verify that they are positive. The pseudomoduli $\phi$ are

$$
\phi \equiv\left(z_{3}, \delta z_{4}, y_{4}^{S}, y_{4}^{A}, x_{14}^{A}, x_{24}^{A}, x_{34}^{A}, x_{34}^{S}\right)
$$

with $z_{4}=\sqrt{F}+\delta z_{4}$. As a check on our technique, we have not parameterized away the Goldstone bosons and instead verify that they remain massless when we compute the mass matrix for $\phi$. 
The one-loop Coleman-Weinberg potential is [23]

$$
V_{C W}=\frac{1}{64 \pi^{2}} \mathrm{~S} \operatorname{Tr}\left[\mathcal{M}^{4} \log \frac{\mathcal{M}^{2}}{\Lambda^{2}}\right]
$$

and the mass matrices are

$$
\mathcal{M}_{B}^{2}=\left(\begin{array}{cc}
\mathcal{M}_{F}^{*} \mathcal{M}_{F} & \mathcal{F}^{*} \\
\mathcal{F} & \mathcal{M}_{F} \mathcal{M}_{F}^{*}
\end{array}\right), \quad \mathcal{M}_{F}^{2}=\left(\begin{array}{cc}
\mathcal{M}_{F}^{*} \mathcal{M}_{F} & 0 \\
0 & \mathcal{M}_{F} \mathcal{M}_{F}^{*}
\end{array}\right)
$$

where $\mathcal{F}_{i j} \equiv F_{k}^{*} W_{i j k}$ and $\mathcal{M}_{F}=W_{i j}$. Because of the relatively large number of fields in the theory, it is not straightforward to compute the eigenvalues of $\mathcal{M}_{B}^{2}$ and $\mathcal{M}_{F}^{2}$ as functions of $\phi$.

It is therefore useful to have a simple numerical technique to calculate the masses. For both fermion and scalar mass matrices, we expand $\mathcal{M}^{2}$ to quadratic order in the $\phi$ fields:

$$
\mathcal{M}^{2}=\max \left(\mathcal{M}^{2}\left(\phi_{0}\right)\right)\left[\mathrm{I}+\delta \mathcal{M}^{2}(\phi)\right]+\mathcal{O}\left(\phi^{3}\right)
$$

where $\phi_{0}$ is the vacuum and $\max \left(\mathcal{M}^{2}\left(\phi_{0}\right)\right)$ is the maximum eigenvalue of $\mathcal{M}^{2}\left(\phi_{0}\right)$. Thus $\max \left(\mathcal{M}^{2}\left(\phi_{0}\right)\right)$ is simply a number and the matrix $\delta \mathcal{M}^{2}(\phi)$ is a quadratic function of the fields $\phi$. Dropping the $\mathcal{O}\left(\phi^{3}\right)$ terms in $\mathcal{M}^{2}$ (because they will not be important for the masses), $V_{C W}$ can be expanded as

$$
V_{C W}=\frac{1}{64 \pi^{2}} \mathrm{~S} \operatorname{Tr}\left[\mathcal{M}^{4} \log \frac{\max \left(\mathcal{M}^{2}\left(\phi_{0}\right)\right)}{\Lambda^{2}}+\sum_{n} \frac{(-1)^{n}}{n} \mathcal{M}^{4}\left(\delta \mathcal{M}^{2}(\phi)\right)^{n}\right]
$$

which is a straightforward calculation of products and traces of matrices. The sum can be truncated at some order $n$, given a desired numerical accuracy.

The pseudomoduli mass matrix is:

$$
m_{\phi}^{2}=\left(\begin{array}{ll}
\frac{\partial^{2} V_{C W}}{\partial \phi \partial \bar{\phi}} & \frac{\partial^{2} V_{C W}}{\partial \phi \partial \phi} \\
\frac{\partial^{2} V_{C W}}{\partial \bar{\phi} \partial \bar{\phi}} & \frac{\partial^{2} V_{C W}}{\partial \phi \partial \bar{\phi}}
\end{array}\right)_{\phi=\phi_{0}}
$$

We have calculated the mass eigenvalues for the pseudomoduli up to $n=20$, and verified the convergence of the series expansion, as shown in Fig. 1. There are always 3 massless directions:

$$
\operatorname{Im}\left(\bar{y}_{4}^{A}+z_{3}\right), \operatorname{Re}\left(y_{4}^{A}+\bar{z}_{3}\right), \operatorname{Im}\left(\delta z_{4}\right)
$$

which are Goldstone bosons. The other 13 fields are true pseudomoduli and have positive mass-squared values, which converge to the numerical values of

$$
m_{i}^{2}=\left|h^{4} F\right|(8,4,4,4,4,2,2,2,2,1,1,1,1) \times \frac{\log 4-1}{4 \pi^{2}} .
$$




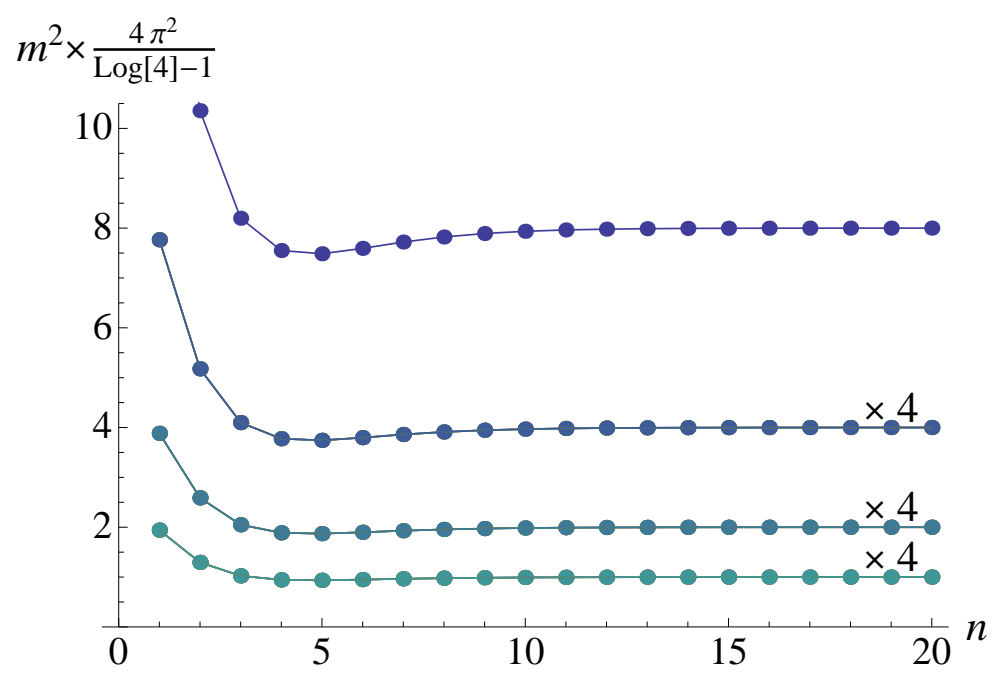

FIG. 1. Pseudomoduli masses of Theory 8, in units of $\left|h^{4} F\right|$, calculated to $n$th order in Eq. 22. The multiplicity of the lower three eigenvalues of indicated.

The structure of the problem is quite rigid; the eigenvectors are the same at every order in the series expansion, as well as the ratios of the eigenvalues. This seems to suggest there might be an analytic way of resumming the series, as the potential may decouple into several sectors as in [14], but we do not pursue this idea further.

\section{B. Higgsing pseudomoduli}

The light fields $P_{1,2}$ appear only in $\Lambda$-suppressed terms in $W$. The leading contribution to their masses, arising from both the Kähler potential, $K$, and the superpotential, are of order $\mathcal{O}\left(|h F / \Lambda|^{2}\right)$ and are thus incalculable without some other stabilization mechanism.

However, if $P_{1}$ and $P_{2}$ have gauge interactions, they can feel supersymmetry breaking more directly if the messengers are also charged under the same gauge symmetry $G$. In this case, the relevant term in the superpotential is

$$
W \supset 2 x_{34}^{S}\left(F-z_{1} y_{2}^{A}+z_{2} y_{1}^{A}\right)
$$

so the messengers are $z_{1,2}$ and $y_{1,2}^{A}$. If the superpartner to the goldstino, $x_{34}^{S}$, is neutral under $G$, then $P_{1,2}$ fall into the class of "Higgsing pseudomoduli" discussed in [24]. The potential for $P_{1}$ and $P_{2}$ is lifted at the two-loop level, at field values $|\sqrt{h F}| \ll\left|P_{1,2}\right| \ll \Lambda$. While the masses of these pseudomoduli are unknown at the origin, the fields are stabilized somewhere 
below $\Lambda$.

The simplest candidate for $G$ is an anomaly-free subgroup of the unbroken flavor symmetry of this model. For our purposes it is sufficient to gauge the $U(1)$ associated with the generator

$$
a \sigma_{z}^{2}+b \sigma_{z}^{U}+c \sigma_{z}^{D}
$$

Here $\sigma_{z}^{2}$ is a generator of $S U(2)_{2}$ and $\left(\sigma_{z}^{U}, \sigma_{z}^{D}\right) \subset S U(2)^{U} \times S U(2)^{D} \subset S p(4)$. This $U(1)$ is anomaly-free for any choice of the coefficients $a, b, c$ above. The charges of $P_{1}$ and $P_{2}$ are $q\left(P_{2}\right)= \pm a$ and $q\left(P_{1}\right)= \pm a \pm b, \pm a \pm c$. The charges of the messengers are $q\left(z_{1,2}\right)= \pm b$ and $q\left(y_{1,2}^{A}\right)= \pm b$, and $x_{34}^{S}$ is neutral. Therefore the $U(1)$ above satisfies the conditions for "Higgsing pseudomoduli" as long as $a$ and $b$ are nonzero, such that $P_{2}$ and the messengers are charged. Note that gauging the $U(1)$ above also justifies the previous statement that the perturbation $J A \bar{Q}^{2} \sim x_{34}^{S}+x_{12}^{S}$ is the lowest dimension operator consistent with gaugeinvariance that we could have added to $W$. The four components $x_{34}^{A, S}, x_{12}^{A, S}$ of the $X$ field are neutral; the $S U(2)_{1}$ symmetry allows us to restrict to only the fields $x_{34}^{S}, x_{12}^{S}$.

We now review the argument of [24] that Higgsing pseudomoduli receive a 2-loop lifting of the superpotential away from the origin. Suppose $\left|P_{1,2}\right| \gg|\sqrt{h F}|$, such that the effects of supersymmetry breaking are small. Then the $U(1)$ gauge boson gets a mass

$$
\frac{m_{A}^{2}\left(\left|P_{1}\right|^{2},\left|P_{2}\right|^{2}\right)}{g^{2}}=\sum_{i} q\left(P_{1}^{i}\right)^{2}\left|P_{1}^{i}\right|^{2}+\sum_{i} q\left(P_{2}^{i}\right)^{2}\left|P_{2}^{i}\right|^{2},
$$

summing over all components of $P_{1}$ and $P_{2}$, and $g$ is the gauge boson coupling. The gauge boson contributes to the anomalous dimensions of the messengers, which include the term $-2 b^{2} \frac{g^{2}}{16 \pi^{2}} \subset \gamma$ above the scale $m_{A}$.

Because $x_{34}^{S}$ couples to the messengers, this introduces a discontinuity of the $x_{34}^{S}$ anomalous dimension at the two-loop level, which then generates the following effective potential in the leading log approximation:

$$
V_{e f f}\left(P_{1,2}\right) \approx 8 b^{2}|h F|^{2} \frac{h^{2} g^{2}}{\left(16 \pi^{2}\right)^{2}} \ln ^{2}\left(\frac{m_{A}^{2}\left(\left|P_{1}\right|^{2},\left|P_{2}\right|^{2}\right)}{|h F|}\right) .
$$

This lifts the $P_{1,2}$ directions $^{1}$.

\footnotetext{
${ }^{1}$ The quadratic part of the superpotential is invariant under a charge conjugation symmetry under which $z_{1} \leftrightarrow y_{2}^{A}, z_{2} \leftrightarrow y_{1}^{A}, x_{13}^{S} \leftrightarrow x_{24}^{S}$, and $x_{23}^{S} \leftrightarrow x_{14}^{S}$. This indicates that there is no one-loop D-term contribution to the $P_{1,2}$ masses. There will be contributions at higher loop, but these terms will be subdominant in the leading log approximation.
} 


\section{Metastability}

The vacuum above was the lowest energy vacuum near the origin. In general, the $\Lambda$ suppressed terms in $W$ could introduce a supersymmetric vacuum somewhere in the moduli space. In fact, it is possible to show that supersymmetry is still broken if these terms are included; one way to see this is that the R-symmetry remains unbroken. (See [18] for a similar analysis of the full superpotential plus tree-level operators.) We have confirmed there are no solutions to the the F-term equations, for the single gauge invariant perturbation considered here, by again computing the Gröbner basis. There could be higher dimension terms in $W$ as well, but these would be suppressed by more powers of $M_{*}$ and generically don't restore supersymmetry at scales below $\Lambda$.

Other local minima may exist at field values at around $\Lambda$, though their vacuum energy is not calculable. If vacua with lower energy exist, then the vacuum at the origin is metastable and can decay. The decay rate can be computed by approximating the potential as a square barrier; then one can use the results of [25]. Here the decay rate is $\propto \exp (-B)$, with

$$
B \sim \frac{(\Delta \phi)^{4}}{V_{0}} \sim \frac{\Lambda^{4}}{|h F|^{2}}
$$

The decay rate is exponentially suppressed for $|\lambda| \sim\left|\frac{\Lambda^{2}}{h F}\right| \ll 1$, which is precisely the limit in which our analysis is valid.

\section{THEORY 7 - SU(5) WITH $3($ 日 $+\bar{\square})$}

$S U(5)$ with one generation (consisting of an antisymmetric tensor and an antifundamental) was analyzed in [17, 26], and $S U(5)$ with two generations was first analyzed in [27]. Both cases exhibited supersymmetry breaking when perturbations to $W$ were added. To our knowledge, there has been no analysis of supersymmetry breaking in an $S U(5)$ gauge theory with 3 generations, i.e., theory 7 . With the aid of s-confinement, we now argue that this theory can have non-supersymmetric vacua after including tree-level perturbations to the superpotential.

Theory 7 has gauge group and matter content: $S U(5)$ with $3(\theta+\bar{\nabla})$, where $\Theta$ is an antisymmetric tensor, $A$, and $\bar{\square}$ is an antifundamental, $\bar{Q}$. When $W=0$ the theory has an $S U(3)_{1} \times S U(3)_{2} \times U(1) \times U(1)_{R}$ anomaly free global symmetry. The strongly coupled 


\begin{tabular}{c|c|cccc} 
& $S U(5)$ & $S U(3)_{1}$ & $S U(3)_{2}$ & $U(1)$ & $U(1)_{R}$ \\
\hline$A$ & $\boxminus$ & $\square$ & 1 & 1 & 0 \\
$\bar{Q}$ & $\square$ & 1 & $\square$ & -3 & $\frac{2}{3}$ \\
\hline \hline$X=A \bar{Q}^{2}$ & 1 & $\square$ & $\square$ & -5 & $\frac{4}{3}$ \\
$Y=A^{3} \bar{Q}$ & 1 & $\square$ & $\square$ & 0 & $\frac{2}{3}$ \\
$Z=A^{5}$ & 1 & $\square$ & 1 & 5 & 0
\end{tabular}

TABLE III. The matter content and symmetries of theory 7, an $S U(5)$ gauge theory. $A$ and $\bar{Q}$ are the microscopic degrees of freedom, and the other fields are the composites of the IR theory.

theory has an s-confined description in terms of the three gauge invariants: $X=A \bar{Q}^{2}, Y=$ $A^{3} \bar{Q}, Z=A^{5}$, with a dynamical superpotential [16]

$$
W^{(d y n)}=X Y Z-\frac{20}{9} Y^{3}
$$

The factor $\frac{20}{9}$ is determined by the classical constraint.

We consider the effect of adding the three lowest dimension gauge invariant operators to the superpotential:

$$
\delta W=\lambda_{I}^{(1) i}\left[A \bar{Q}^{2}\right]_{i}^{I}+\lambda_{a i}^{(2)} \frac{\left[A^{3} \bar{Q}\right]^{a i}}{M_{*}}+\lambda_{I J}^{(3)} \frac{\left[A^{5}\right]^{I J}}{M_{*}^{2}}
$$

where $I(a)$ is a fundamental (adjoint) index in $S U(3)_{1}$ and $i$ is a fundamental index in $S U(3)_{2}$. For generic values of $\lambda^{(1),(2),(3)}$ one can use the techniques of [28] to show that these perturbations lift all D-flat directions.

We will take $\frac{\Lambda}{M_{*}}=\eta \ll \lambda^{(1)} \sim \lambda^{(2)} \sim \lambda^{(3)} \ll 1$, which is a natural hierarchy when $\Lambda$ is generated by dimensional transmutation. In this limit, these interactions can be treated as perturbations of the $W=0$ theory. We first consider the effect of only the $\lambda^{(1)} A \bar{Q}^{2}$ operator because it is the most important operator near the origin of field space. Afterwards, we will address the effect of $\lambda^{(2),(3)} \neq 0$.

An $S U(3)_{1}$ and $S U(3)_{2}$ flavor rotation can bring $\lambda_{I}^{(1) i}$ into diagonal form. For simplicity, 
we assume in what follows that $\lambda^{(1)}$ preserves the diagonal $S U(3)_{D} \subset S U(3)_{1} \times S U(3)_{2}$,

$$
\lambda^{(1)}=\lambda\left(\begin{array}{lll}
1 & 0 & 0 \\
0 & 1 & 0 \\
0 & 0 & 1
\end{array}\right) \text {. }
$$

After rescaling the fields to obtain canonical kinetic terms, as described in Section II, the full "s-confined" superpotential becomes

$$
W=h_{2}\left(\frac{h_{1}}{h_{2}} X_{i}^{I} Y^{a i} T_{K}^{a J} Z^{K L} \epsilon_{I J L}+\epsilon_{i j k} f^{a b c} Y^{a i} Y^{b j} Y^{c k}+F \delta_{I}^{i} X_{i}^{I}\right)
$$

where $h_{1}=\sqrt{\alpha_{X} \alpha_{Y} \alpha_{Z}}$ and $h_{2}=-\frac{20}{9} \alpha_{Y}^{\frac{3}{2}}$ are unknown dimensionless couplings; and $h_{2} F=$ $\sqrt{\alpha_{X}} \lambda \Lambda^{2} \ll \Lambda^{2}$. The Yukawa couplings $h_{1}$ and $h_{2}$ are marginally irrelevant. The ratio of the couplings $r=\frac{h_{1}}{h_{2}}$ renormalizes to a fixed point in the infrared: $\beta(r)=\frac{\left(h_{2}\right)^{2} r}{32 \pi^{2}}\left(-27+7 r^{2}\right)$ with two stable fixed points, $\beta\left(r_{*}\right)=0$, at $r_{*}= \pm 3 \sqrt{\frac{3}{7}}$. Both interactions in the superpotential are important for the IR physics.

The O'Raifeartaigh model of Eq. (34) can be shown to have no supersymmetric vacua at finite values for the field VEVs. We have verified this by using the Gröbner basis algorithm in Mathematica, as described in Section II. If the Gröbner basis algorithm returns a constant as an element of the generating set, then there is no solution at finite field values. As in the previous section, we find the minimum reduction of the full perturbation, such that there are solutions. In this case, the system of equations has solutions if the rank of the matrix $\lambda^{(1)}$ is reduced by one. An example of such a solution is

$$
\begin{gathered}
\frac{Y^{a k} T_{J}^{a I}}{\sqrt{F}}=\left[\left(\begin{array}{ccc}
0 & \frac{1}{\sqrt{2}} & 0 \\
\frac{1}{\sqrt{2}} & 0 & -1 \\
0 & -1 & 0
\end{array}\right),\left(\begin{array}{ccc}
\frac{1}{\sqrt{2}} & 0 & 1 \\
0 & -\frac{1}{\sqrt{2}} & 0 \\
1 & 0 & 0
\end{array}\right), 0\right] \\
X_{i}^{I}=0, \\
\frac{Z^{I J}}{\sqrt{F}}=\frac{1}{r_{*}}\left(\begin{array}{lll}
0 & 0 & 0 \\
0 & 0 & 0 \\
0 & 0 & 1
\end{array}\right)
\end{gathered}
$$

In the theory with a full rank $\lambda^{(1)}$ this vacuum breaks supersymmetry, but is classically 
unstable due to the presence of a runaway direction. This direction is parameterized by

$$
\begin{aligned}
\frac{Y^{a k} T_{J}^{a I}}{\sqrt{F}}= & {\left[\left(\begin{array}{ccc}
0 & \frac{1}{\sqrt{2}} & 0 \\
\frac{1}{\sqrt{2}} & 0 & -1 \\
0 & -1 & 0
\end{array}\right),\left(\begin{array}{ccc}
\frac{1}{\sqrt{2}} & 0 & 1 \\
0 & -\frac{1}{\sqrt{2}} & 0 \\
1 & 0 & 0
\end{array}\right),\left(\begin{array}{ccc}
\frac{\epsilon}{6} & \frac{\epsilon}{2} & 0 \\
\frac{\epsilon}{2} & \frac{\epsilon}{6} & 0 \\
0 & 0 & -\frac{\epsilon}{3}
\end{array}\right)\right], } \\
X_{i}^{I}=0, & \frac{Z^{I J}}{\sqrt{F}}=\frac{1}{r_{*}}\left(\begin{array}{ccc}
\frac{1}{\epsilon} & \frac{1}{\epsilon} & \frac{\sqrt{2}}{\epsilon} \\
\frac{1}{\epsilon} & -\frac{1}{\epsilon} & -\frac{\sqrt{2}}{\epsilon} \\
\frac{\sqrt{2}}{\epsilon} & -\frac{\sqrt{2}}{\epsilon} & 1
\end{array}\right) .
\end{aligned}
$$

The vacuum energy is $V \sim|\epsilon F|^{2}$, vanishing in the limit that $\epsilon \rightarrow 0$. Therefore the theory has a runaway direction in the $Z$-fields.

Near the origin, the effects of the $\lambda^{(2)}$ and $\lambda^{(3)}$ terms can be neglected. When $\langle Z\rangle \gg \Lambda$, it is more convenient to describe the theory in terms of elementary fields. In this case, the $\lambda^{(2)}$ and $\lambda^{(3)}$ terms become important and the vacuum energy rises with the VEV of the $Z$-field, as discussed earlier. We can then conclude that a VEV for some field must develop around

the scale $\Lambda$. A careful analysis reveals that this theory possesses an approximate R-symmetry where $R(X)=2, R(Y)=-R(Z)=\frac{2}{3}$. Non-zero values for $\lambda^{(2)}$ and $\lambda^{(3)}$ introduce a small explicit breaking of this R-symmetry. This model then falls into the classification of [29]. On the runaway direction, or for that matter anywhere except the origin, this approximate Rsymmetry becomes spontaneously broken since all fields carry R charge. Therefore, we may conclude this theory spontaneously breaks supersymmetry and also spontaneously breaks an approximate R-symmetry at field values of $\mathcal{O}(\Lambda)$. However, the details of this vacuum state are not calculable due to our ignorance of the Kähler potential.

\section{OTHER $S U(N)$ S-CONFINING THEORIES}

In this section we briefly describe perturbations to the other $S U(N)$ s-confining theories, focusing on the region of the moduli space near the origin. After canonically normalizing the fields, we can neglect $\Lambda$-suppressed interactions in $W$. This restricts the interacting field content to fields that have relevant interactions. We will only consider perturbing the $W=0$ theories with a single lowest dimension operator consistent with gauge invariance. The other $S U(N)$ theories generally fall into several classes in this context. Theories 2, 3 and 5 do not have supersymmetry breaking for the simplest linear perturbation. There are 
simple perturbations to the other theories which break supersymmetry. However, theories 4, 6, and 10 possess irrelevantly coupled pseudomoduli, which we find impossible to stabilize with a gauged flavor symmetry. Meanwhile, theory 9 has a runaway in the tree-level fields, similar to theory 7. As we discussed in detail in Section III, these theories generally have supersymmetry-breaking vacua at field values of $\mathcal{O}(\Lambda)$, but there is no small parameter with which to control the calculation there.

In what follows, we omit $\mathcal{O}(1)$ factors and flavor indices for simplicity and assume that kinetic terms have all been canonically normalized.

\section{A. $\quad$ Theory $2-S U(2 N)$ with $\boxminus+2 N \bar{\square}+4 \square$}

Theory 2 is an $S U(2 N)$ gauge theory with an antisymmetric tensor $\boxminus(A), 2 N$ antifundamentals $\bar{\square}(\bar{Q})$, and 4 fundamentals $\square(Q)$.

1. $\quad N>2$

For $N>2$, the IR theory has 3 singlets $X=A^{N}, Y=A^{N-2} Q^{4}, Z=\bar{Q}^{2 N}$ and an antisymmetric tensor under the $S U(4)$ flavor symmetry: $\Phi_{i}=A^{N-1} Q^{2}$, where we have written $\Phi_{i}$ as a 6 -vector for convenience. The lowest dimension perturbation that can be added is $\delta W=h F X$. Because $X$ is a singlet under the non-Abelian flavor symmetries, we cannot invoke a gauge symmetry that forbids this operator but also permits other perturbations. Thus the leading order superpotential is

$$
W=h(X Y Z+Z \vec{\Phi} \cdot \vec{\Phi}+F X+\ldots)
$$

In this theory, supersymmetry is unbroken.

We note that supersymmetry is broken if we also include the next lowest dimension perturbation, such that $\delta W=h(F X+\lambda F \vec{v} \cdot \vec{\Phi})$, with $\vec{v}=(0,0,0,0,0,1)$. However, this theory possesses a runaway direction defined by, as $\epsilon \rightarrow 0$,

$$
X=0, Y=\sqrt{F} \epsilon, Z=-\frac{\sqrt{F}}{\epsilon}, \Phi=\lambda \sqrt{F} \epsilon \vec{v}
$$




\section{2. $N=2$}

In the $N=2$ case, the theory has a larger flavor symmetry, $S U(4)_{1} \times S U(4)_{2}$, and an extra relevantly-coupled field $\chi_{i}=A \bar{Q}^{2}$, an antisymmetric tensor under the $S U(4)_{2}$ flavor symmetry. As in the $N>2$ case, the most general lowest-dimension operator we can add is $\delta W=h F X$, and supersymmetry is unbroken.

We could consider higher order perturbations,

$$
W=h\left(X Y Z+Z \vec{\Phi} \cdot \vec{\Phi}+Y \vec{\chi} \cdot \vec{\chi}+F X+\lambda^{(1)} F \vec{v} \cdot \vec{\Phi}+\lambda^{(2)} F \vec{v}^{\prime} \cdot \vec{\chi}+\ldots\right) .
$$

Supersymmetry is still unbroken unless one of $\lambda^{(1,2)}$ is zero; one can imagine doing this by weakly gauging a subgroup of one of the $S U(4)$ flavor symmetries. Then supersymmetry is broken, but there is a runaway direction. For the case $\lambda^{(2)}=0$, the runaway direction is given by Eq. 40, plus the condition $\chi=0$.

\section{B. Theory $3-S U(2 N+1)$ with $日+(2 N+1) \square+4 \square$}

The IR theory near the origin has an $S U(4)$ flavor symmetry, with an $\square\left(X^{i}\right)$, an $\bar{\square}\left(Y_{i}\right)$, and a singlet $Z$. The lowest-order perturbed superpotential is

$$
W=h(\vec{X} \cdot \vec{Y} Z+F \vec{v} \cdot \vec{X})
$$

where $\vec{v}=(0,0,0,1)$. Supersymmetry is unbroken in this case.

If the next order perturbation $\delta W=h \lambda F \vec{v} \cdot \vec{Y}$ is also included, then supersymmetry is broken. However, there is a runaway direction: $Z \rightarrow \frac{\sqrt{F}}{\epsilon}, \vec{Y} \rightarrow-\sqrt{F} \epsilon \vec{v}, \vec{X} \rightarrow-\lambda \sqrt{F} \epsilon \vec{v}$.

\section{Theory $4-S U(2 N+1)$ with $\boxminus+\bar{\theta}+3(\square+\bar{\square}), N=2$}

The full theory is $S U(2 N+1)$ with $\boxminus+\overline{\bar{\theta}}+3(\square+\bar{\square})$. For simplicity we will only consider the $N=2$ case. The lowest-dimension perturbation is $\delta W=h F M_{0}$. Then then superpotential contains the terms

$$
W \supset h\left(\left(H_{1}\right)_{a}\left(\bar{H}_{1}\right)_{\bar{b}} M_{0}^{a \bar{b}}+F d_{a \bar{b}} M_{0}^{a \bar{b}}\right)
$$

where $a, \bar{b}$ are $S U(3)_{1}, S U(3)_{2}$ flavor indices and $M_{0}$ appears in no other terms in $W$. Thus this looks like an example of rank-breaking. However, this theory contains a number of 
flavor singlets $T$ which are only irrelevantly coupled, so the stability of the vacuum near the origin is incalculable.

D. Theory $5-S U(2 N)$ with $\boxminus+\bar{\theta}+3(\square+\bar{\square}), N=2$

The theory is $S U(2 N)$ with $\boxminus+\overline{\bar{G}}+3(\square+\bar{\square})$, with flavor symmetry $S U(2) \times S U(3)_{1} \times$ $S U(3)_{2}$. Only the $N=2$ superpotential is given in [16]. The superpotential with the lowest dimension perturbation is

$$
W=h\left(M_{0}\left(M_{2}\right)^{2}+H \bar{H} M_{2}+F J M_{0}\right)
$$

Here $H_{a}^{i}, \bar{H}_{\bar{a}}^{i}$ transform under $i \in S U(2), a \in S U(3)_{1}, \bar{a} \in S U(3)_{2}$ while $\left(M_{0}\right)^{a \bar{b}}$ and $\left(M_{2}\right)^{a \bar{b}}$ transform under $S U(3)_{1} \times S U(3)_{2}$. This theory has supersymmetric vacua.

\section{E. Theory $6-S U(6)$ with $\theta+4(\square+\bar{\square})$}

This theory was recently investigated in [19]. The theory has an $S U(4)_{1} \times S U(4)_{2}$ flavor symmetry. The lowest-dimension perturbation is $\delta W=h F M_{0}$ and supersymmetry is broken via rank breaking:

$$
W \supset h\left(\left(B_{3}\right)_{a}\left(\bar{B}_{3}\right)_{\bar{b}} M_{0}^{a \bar{b}}+F d_{a \bar{b}} M_{0}^{a \bar{b}}\right)
$$

where $a, \bar{b}$ are $S U(4)_{1}, S U(4)_{2}$ flavor indices and $M_{0}$ appears in no other terms in $W$. However, this theory contains a singlet $T$ which is only irrelevantly coupled.

\section{F. Theory $9-S U(6)$ with $2 \boxminus+5 \bar{\square}+\square$}

The theory is $S U(6)$ with $2 \boxminus(A)+5 \bar{\square}(\bar{Q})+\square(Q)$. The IR fields are $M=Q \bar{Q}, X=$ $A \bar{Q}^{2}, Y=A^{3} Q \bar{Q}$, and $Z=A^{4} \bar{Q}^{2}$ and the dynamical superpotential is

$$
W^{(d y n)}=M Z^{2}+X Y Z
$$

with flavor symmetry $S U(2) \times S U(5)$. The lowest dimension perturbation is $\delta W=h F J M$, but the theory does not break supersymmetry. 
We also considered the perturbation $\delta W=h F J X$, where $X^{i a b}$ is an $(\square, \boxminus)$ under $S U(2) \times$ $S U(5)$. The tensor $J$ is given by

$$
J_{i a b}=\frac{1}{\sqrt{2}}\left(\left(\begin{array}{ccccc}
0 & 1 & 0 & 0 & 0 \\
-1 & 0 & 0 & 0 & 0 \\
0 & 0 & 0 & 1 & 0 \\
0 & 0 & -1 & 0 & 0 \\
0 & 0 & 0 & 0 & 0
\end{array}\right),\left(\begin{array}{ccccc}
0 & 1 & 0 & 0 & 0 \\
-1 & 0 & 0 & 0 & 0 \\
0 & 0 & 0 & 1 & 0 \\
0 & 0 & -1 & 0 & 0 \\
0 & 0 & 0 & 0 & 0
\end{array}\right)\right) \text {. }
$$

The perturbation is invariant under $S p(4) \times U(1) \in S U(5)$; weakly gauging this symmetry forbids the lower-dimension perturbation $\delta W=h F J M$. This theory breaks supersymmetry, but not via rank-breaking. We did not find a classically stable vacuum near the origin and we expect there to be a runaway direction, analogous to theory 7 .

\section{G. Theory $10-S U(7)$ with 2 日 $+6 \bar{\square}$}

This theory has already been discussed in [16]. The theory is $S U(7)$ with $2 \boxminus(A)+6 \bar{\square}(\bar{Q})$ and has IR degrees of freedom $H=A \bar{Q}^{2}, N=A^{4} \bar{Q}$. The superpotential is $W^{(d y n)}=$ $N^{2} H^{2} / \Lambda$, so there are no relevant interactions. It is possible that this theory breaks supersymmetry if a perturbation in the $H$ fields is added. However, there is no calculable vacuum near the origin.

\section{CONCLUSIONS}

Perturbations to $\mathcal{N}=1$ supersymmetric gauge theories can give rise to dynamical supersymmetry breaking. We have considered the "s-confining" $S U(N)$ gauge theories of [16] and added to the superpotential a single gauge-invariant operator. These models are described by a relatively complicated O'Raifeartaigh model near the origin of the moduli space. We have employed two methods for analyzing the vacuum structure. First, in order to solve the complicated F-term equations that arise, it is useful to compute the Gröbner basis. This also gives a straightforward way of showing that supersymmetry is broken at finite field values. Second, because of the large number of fields, we have calculated 1-loop pseudomoduli masses numerically in a series expansion. We have shown that the series converges for our example. 
In our first model, we added a perturbation to theory 8, an $S U(5)$ gauge theory with two generations of an antisymmetric tensor plus antifundamental and two flavors. Here we found a locally stable and calculable vacuum with supersymmetry breaking near the origin of moduli space. However, we were required to gauge an anomaly-free subset of the global flavor symmetry. This ensured the stability of the irrelevantly coupled pseudo-moduli, and justified our choice of perturbation as the lowest dimension operator allowed by gauge invariance.

The second example we gave was theory 7, an $S U(5)$ gauge theory with 3 generations of an antisymmetric tensor plus antifundamental. Our analysis adds a new chapter to the history of $S U(5)$ models as models of dynamical supersymmetry breaking. We found that adding the lowest dimension gauge invariant operator removes any supersymmetric vacuum from the theory. Near the origin, the framework of s-confinement can be used to show that there is a runaway direction to large field VEVs, at which point the elementary description becomes valid. If there are other higher dimensional operators, which can be neglected for the analysis near the origin, then all flat directions can be lifted at large field values. The theory will stabilize in the incalculable region with field VEVs of order $\mathcal{O}(\Lambda)$. Furthermore, because we know that all fields carry R-charge, the accidental R-symmetry is spontaneously broken.

We also briefly considered other $S U(N)$ s-confining theories and found that, while they do have rich and interesting dynamics, they either 1) do not lead to supersymmetry breaking when a single lowest dimension operator is added to the theory, 2) they possess irrelevantly coupled flavor singlets and cannot be rendered calculable by gauging a flavor symmetry, or 3) they have tree level runaways to large field VEVs, as in theory 7. In the latter two cases, any supersymmetry breaking vacuum will generally be in an incalculable regime.

\section{ACKNOWLEDGMENTS}

We are grateful for many useful conversations with Frederik Denef, Howard Georgi, Zohar Komargodski, Vijay Kumar, David Poland, Martin Schmaltz, Nathan Seiberg, Yael Shadmi, David Shih, Yuri Shirman, and David Simmons-Duffin. We thank Vijay Kumar and Yael Shadmi for helpful comments on the paper. JM would like thank the Aspen Center for

Physics for their hospitality while this work was being done as well as the organizers of the 
SUSY Breaking 2011 Workshop. This research was supported by NSF Grant PHY-0855591.

[1] E. Witten, Nucl.Phys. B188, 513 (1981).

[2] I. Affleck, M. Dine, and N. Seiberg, Phys.Rev.Lett. 51, 1026 (1983).

[3] I. Affleck, M. Dine, and N. Seiberg, Nucl.Phys. B241, 493 (1984).

[4] N. Seiberg, Phys.Lett. B318, 469 (1993), arXiv:hep-ph/9309335.

[5] N. Seiberg, Phys.Rev. D49, 6857 (1994), arXiv:hep-th/9402044.

[6] N. Seiberg, Nucl.Phys. B435, 129 (1995), arXiv:hep-th/9411149.

[7] E. Poppitz and S. P. Trivedi, Phys.Lett. B365, 125 (1996), arXiv:hep-th/9507169, Revised version.

[8] E. Poppitz, Y. Shadmi, and S. P. Trivedi, Nucl.Phys. B480, 125 (1996), arXiv:hep-th/9605113.

[9] P. Pouliot, Phys.Lett. B367, 151 (1996), arXiv:hep-th/9510148.

[10] J. Terning, Phys.Lett. B422, 149 (1998), arXiv:hep-th/9712167.

[11] N. Craig, R. Essig, A. Hook, and G. Torroba, arXiv:1106.5051, arXiv:1106.5051 [hep-th].

[12] L. O'Raifeartaigh, Nucl.Phys. B96, 331 (1975).

[13] A. E. Nelson and N. Seiberg, Nucl.Phys. B416, 46 (1994), arXiv:hep-ph/9309299.

[14] K. A. Intriligator, N. Seiberg, and D. Shih, JHEP 0604, 021 (2006), arXiv:hep-th/0602239.

[15] C. Csaki, M. Schmaltz, and W. Skiba, Phys.Rev.Lett. 78, 799 (1997), arXiv:hep-th/9610139.

[16] C. Csaki, M. Schmaltz, and W. Skiba, Phys.Rev. D55, 7840 (1997), arXiv:hep-th/9612207.

[17] H. Murayama, Phys.Lett. B355, 187 (1995), arXiv:hep-th/9505082.

[18] T. A. ter Veldhuis, Phys.Rev. D58, 015010 (1998), arXiv:hep-th/9802125.

[19] Y. Shadmi, (2011), To appear.

[20] Y. Shadmi and Y. Shirman, To appear: see Y. Shadmi's talk at 'SUSY Breaking 2011'.

[21] B. Buchberger, SIGSAM Bull. 10, 19 (1976).

[22] D. Cox, J. Little, and D. O'Shea, Ideals, Varieties, and AlgorithmsUndergraduate Texts in Mathematics (Springer-Verlag, New York, 1997).

[23] S. R. Coleman, Phys.Rev. D15, 2929 (1977).

[24] K. Intriligator, D. Shih, and M. Sudano, JHEP 0903, 106 (2009), arXiv:0809.3981.

[25] M. J. Duncan and L. G. Jensen, Phys.Lett. B291, 109 (1992).

[26] I. Affleck, M. Dine, and N. Seiberg, Phys.Lett. B137, 187 (1984). 
[27] I. Affleck, M. Dine, and N. Seiberg, Phys.Rev.Lett. 52, 1677 (1984).

[28] M. A. Luty and W. Taylor, Phys.Rev. D53, 3399 (1996), arXiv:hep-th/9506098.

[29] J. Goodman, M. Ibe, Y. Shirman, and F. Yu, (2011), arXiv:1106.1168. 